\title{
Electrically detected magnetic resonance of ion-implantation damage centers in silicon large-scale integrated circuits
}

\author{
T. Umeda ${ }^{\mathrm{a})}$ and Y. Mochizuki ${ }^{\mathrm{b})}$ \\ Silicon Systems Research Laboratories, NEC Corporation, Sagamihara, 229-1198, Japan \\ K. Okonogi and K. Hamada \\ Elpida Memory Incorporation, Sagamihara, 229-1198, Japan
}

(Received 5 May 2003; accepted 15 September 2003)

\begin{abstract}
We used electrically detected magnetic resonance to study the microscopic structure of ion-implantation-induced point defects that remained in large-scale Si integrated circuits (Si LSIs). Two types of defects were detected in the source/drain $\left(n^{+}\right.$-type) region of 0.25 - $\mu \mathrm{m}$-gate-length $n$-channel metal oxide semiconductor field-effect-transistors on LSIs: (i) a spin-1 Si dangling-bond (DB) pair in divacancy-oxygen complexes (DB-DB distance, $R \approx 0.6 \mathrm{~nm}$ ); and (ii) a series of larger $\mathrm{Si}$ vacancies involving distant $\mathrm{Si} \mathrm{DBs}(R \geqslant 1.4 \mathrm{~nm})$. These vacancy-type defects were much more thermally stable in Si LSIs than those in bulk Si crystals. We suggested two physical mechanisms for this enhanced stability: internal mechanical stress and oxygen incorporation in the active regions of LSIs. After examining the relationship between the defects and current-voltage characteristics, we concluded that these defects are distributed in the near-surface $n^{+}$-type region close to the gate and that they are the source of the gate-induced drain leakage currents. (c) 2003 American Institute of Physics. [DOI: 10.1063/1.1623608]
\end{abstract}

\section{INTRODUCTION}

Ion implantation is a key technology in the fabrication of large-scale Si integrated circuits (Si LSIs). This process always requires high-temperature annealing, typically at $1000{ }^{\circ} \mathrm{C}$, to recover the crystalline lattice, annihilate defects, and activate implanted dopants. However, even after recovery annealing, some defects still remain, which is evident from electrical measurements. For large defects such as dislocations, it is possible to obtain information directly by using transmission electron microscopy (TEM) combined with focused-ion-beam processing for LSIs. ${ }^{1}$ However, in studying small point defects, there were no methods that were directly applicable to LSIs. Characterizing these point defects is important because a number of failures in LSIs show no detectable defects in TEM analyses, and are therefore believed to relate to much smaller defects. With the current trend towards downsizing device regions, such defects have become increasingly critical in terms of device performance. Furthermore, the complicated processing steps used for LSIs are resulting in the formation of more process-induced defects.

One possible means of detecting point defects in LSIs is the use of electrically detected magnetic resonance (EDMR) ${ }^{2-5}$ Since this technique monitors the magnetic resonance of defects via resonant changes in the device current, it is applicable to the small sample volumes in LSIs. Moreover, EDMR selectively detects only defects along the current path and thus related to device performance. It can

\footnotetext{
${ }^{a}$ Present address: Research Center for Knowledge Communities, University of Tsukuba, Tsukuba 305-8550, Japan; electronic mail: umeda@slis.tsukuba.ac.jp

b) Author to correspondence should be addressed; electronic mail: y-mochizuki@az.jp.nec.com
}

give us microscopic information about defects equivalent to that obtained using electron paramagnetic resonance (EPR). EPR has greatly improved our understanding of knowledge about bulk-Si defects, ${ }^{6}$ so that EDMR should be equally useful. However, despite the potential of EDMR, its application to Si devices has so far been confined to examining nonintegrated devices such as large-area Si $p-n$ diodes $^{7}$ or damaged devices such as the structure of a metal oxide semiconductor (MOS) after $\gamma$-ray irradiation, ${ }^{8}$ in order to increase the defect signal.

In this paper, we report EDMR measurements on asfabricated $n$-channel metal oxide semiconductor field-effect transistors (MOSFETs) on Si LSIs, which were made using a $0.25-\mu \mathrm{m}$-rule process. The detection sensitivity to defects can be greatly enhanced by using a reverse-bias EDMR method and a carefully designed MOSFET array. These techniques make it possible to identify the microscopic structure of process-induced defects in device-quality samples. The defects observed were two types of $\mathrm{Si}$ vacancies produced by the ion implantation in the source/drain region of the MOSFETs. It was believed that these vacancy-type defects would not remain in Si LSIs because of their low thermal stability in bulk Si crystals. However, it should be emphasized that regions in downsized devices are quite different from those in a normal Si lattice, which makes the defects very stable even at high temperatures $\left(\sim 1000^{\circ} \mathrm{C}\right)$. The physical mechanisms for this enhanced stability are discussed in this paper based on our experimental results. We also examined the relationship between the defects and the $I-V$ characteristics of MOSFETs. Eventually, it was found that these defects relate directly to the gate-induced drain leakage (GIDL) current of MOSFETs. Since the GIDL constrains the scaling of Si LSIs,${ }^{9,10}$ the defects we observed are an important issue in LSI technologies. 
(a)

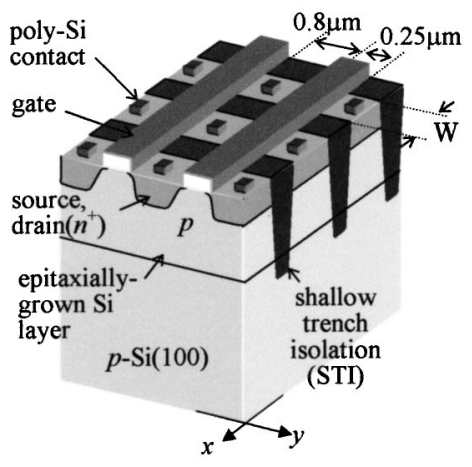

(b)

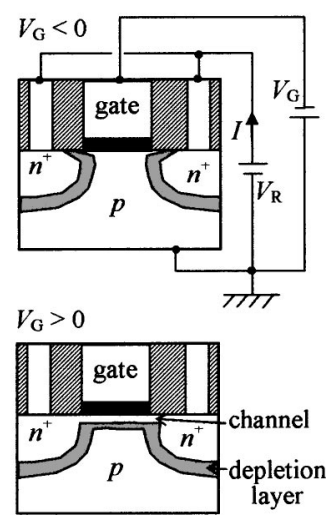

FIG. 1. (a) Sample structure. (b) Electrical configuration for EDMR measurements and schematic views of gate-bias $\left(V_{G}\right)$ dependence of the sample. Gray area represents the depletion layer.

\section{EXPERIMENTAL PROCEDURE}

Figure 1(a) shows the basic structure of the samples we examined, which consisted of a parallel array of $n$-channel MOSFETs with a gate length of $0.25 \mu \mathrm{m}$ and a gate-oxide thickness of $9 \mathrm{~nm}$. The source/drain $\left(n^{+}\right.$-type) region was formed by phosphorous ion implantations with a total dose of about $10^{13} \mathrm{~cm}^{-2}$. Recovery annealing was carried out at $800 \sim 1000{ }^{\circ} \mathrm{C}$. Under these conditions, the TEM images showed no lattice defects. To obtain sufficient signal intensity from the device-quality samples, we integrated a number of the MOSFETs into one sample so that the total source/ drain area increased to $34560 \mu \mathrm{m}^{2}$. The LSI process used in this study was the same as that used for dynamic random access memories (DRAMs), which has been optimized to minimize leakage currents in the $n^{+}-p$ junction (junction leakage currents, JLCs). Therefore, the density of defects has also been minimized in our device regions. The lines of MOSFET along the $y$-direction were isolated by shallow trench isolations (STIs). It is well known that these STIs generate strong mechanical stress in specific device regions, depending on the STI pitch $(W)$. In general, decreasing $W$ causes a strong compressive stress in the MOSFET regions, ${ }^{11,12}$ which frequently results in an appreciable increase in JLCs. ${ }^{12-14}$ This implies that STI-induced stress correlates with the formation of defects. To examine how this stress influences defects and device performance, we prepared three samples with different STI pitches of 24, 2.4, and $0.8 \mu \mathrm{m}$. The existence of the STI-induced stress has been confirmed in a STI structure similar to this one. ${ }^{15}$ The three samples were fabricated on a common 8-in. wafer, so that the process for each one was completely identical. Finally, for EDMR measurements, we cut small-sized chips $(0.5 \times 0.5$ $\mathrm{cm}^{2}$ ) from a wafer and made electrical contacts with Au-wire bonding.

The EDMR spectra were measured with a modified X-band EPR spectrometer (JEOL RE-2X), which we described previously. ${ }^{4,5}$ The sample was set in the center of a $\mathrm{TE}_{011}$ cylindrical microwave cavity $(Q$ factor $=18000)$. Since our samples were very small, we were able to apply a microwave power of $200 \mathrm{~mW}$ to them with a minimum di-

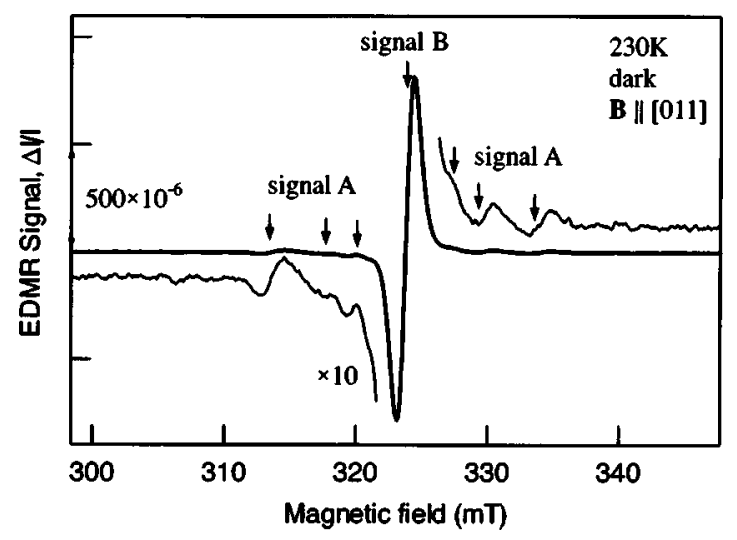

FIG. 2. EDMR spectrum of our sample $(W=0.8 \mu \mathrm{m})$ measured under a reverse-bias condition.

electric loss. Figure 1(b) shows the electrical configuration for the EDMR. The rate of resonant current change $(\Delta I / I)$ was monitored for the current (I) flowing from the source/ drain $\left(n^{+}\right.$-type layer) to the $p$-type substrate of the MOSFETs; in other words, the $n^{+}-p$ junction current was used for the EDMR. To amplify the resonant current changes, we used a magnetic-field-modulation technique operating at 320 Hz. The standard modulation width was $0.9 \mathrm{mT}$, but to enlarge the center part of the spectra, a width of $0.4 \mathrm{mT}$ was used. The measurement temperature was adjusted to $230 \mathrm{~K}$ based on the following considerations. In principle, the spin resonance signal increases with decreasing temperatures, according to Curie's law. However, at low temperatures, thermal generation-recombination processes via defects are largely suppressed, and Si devices no longer show their normal $I-V$ characteristics. Accordingly, an optimum temperature should be established for EDMR measurements, which was $230 \mathrm{~K}$ in this study. To prevent the generation of photoexcited carriers, no illumination was applied.

\section{RESULTS}

\section{A. Detection of defect signals and relationship between the defects and $I-V$ characteristics}

Figure 2 shows a typical EDMR spectrum for our samples. Two EDMR signals, which we called signals A and $\mathrm{B}$, can be clearly observed. This spectrum was measured using two special techniques that greatly increase the signal intensity. Before analyzing the spectrum, we describe the detection conditions for these signals and clarify the relationship between them and the $I-V$ characteristics of our devices.

A key point in the signal detection was the use of a reverse-bias EDMR technique. ${ }^{5}$ Namely, we applied a reverse bias to the $p-n$ junctions for taking the EDMR signals. In previous studies using EDMR, either a forward bias or photoexcitation of the $p-n$ junction has been used to excite a large current. ${ }^{2-4}$ Under such conditions, however, only a small fraction of the defect-induced recombination currents is generated, as compared to diffusion currents. However, reverse junction leakage currents can include a larger fraction of defect-induced currents. ${ }^{16}$ Among the various pro- 


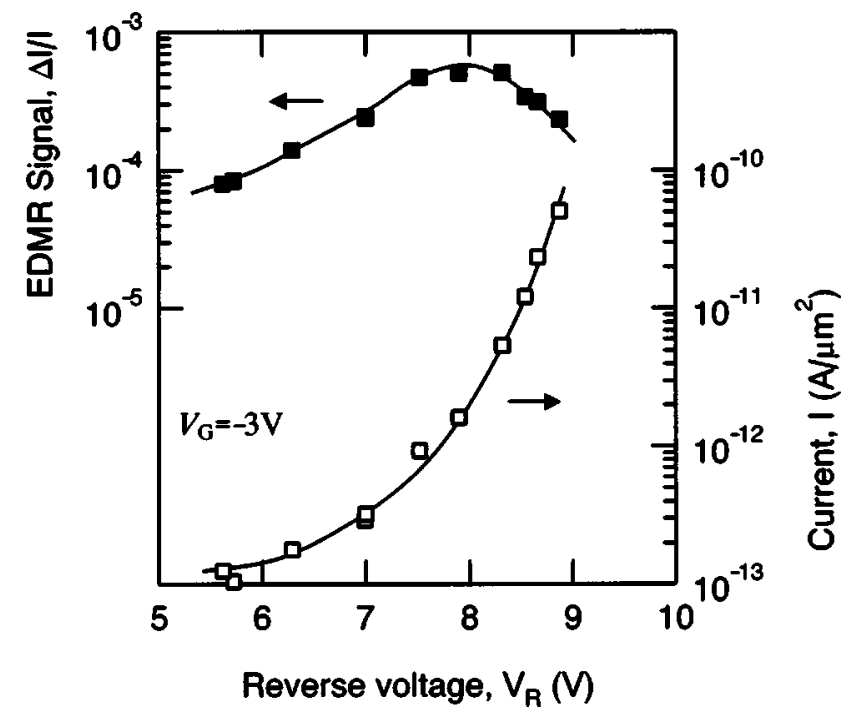

FIG. 3. Reverse-bias dependence of the EDMR signal and device current in a sample $(W=0.8 \mu \mathrm{m})$. The signal intensity $(\Delta I / I)$ is calculated from the higher-field-side peak height of signal B. Solid lines are guides to the eyes.

cesses that generate JLCs, capturing an electron by a paramagnetic defect in the depletion layer is spin dependent. ${ }^{17}$ The probability of this process occurring increases with the reverse bias due to tunneling of electrons from the Si valence band to the defect. ${ }^{18}$ We therefore expected that signal detection would be more successful under a high reverse bias $\left(V_{R}\right)$, and in fact, the EDMR signals increased considerably under these conditions (Fig. 3). When $V_{R}$ is larger than $6 \mathrm{~V}$, the signal intensity $(\Delta I / I)$ easily exceeds $100 \times 10^{-6}$, which is much larger than the maximum value of $\Delta I / I$ for a forward bias $\left(15 \times 10^{-6}\right)$. In addition, in our previous EDMR measurements of a planar Si $p-n$ diode (forward biased, 4 $\mathrm{K}$, and under photoexcitation), the maximum $\Delta I / I$ value was no more than $10 \times 10^{-6} .{ }^{4}$ Therefore, the detection sensitivity was greatly enhanced by using a reverse-bias EDMR method as compared to conventional forward-bias/photoexcitation EDMR. As the reverse bias increased further, the JLC continued to increase, but the $\Delta I / I$ decreased. This implies that direct tunneling of carriers through the depletion layer started to occur. When $V_{R}$ was over $10 \mathrm{~V}$, the electric field of the depletion layer increased to $1 \mathrm{MV} / \mathrm{cm}$. In this regime, the tunneling and avalanche currents become dominant, ${ }^{16,18}$ and eventually the $p-n$ junction undergoes a breakdown.

In addition to using a high reverse bias, controlling the depletion layer with the gate bias is also necessary for signal detection. As shown in Fig. 1(b), changing the gate voltage $\left(V_{G}\right)$ causes the depletion layer to expand into the nearsurface region of either $n^{+}$- or $p$-type Si. Therefore, the gate bias enabled us to focus on the region in which most of the defects were located. Figure 4(a) shows the EDMR signal intensity as a function of $V_{G}$ for the three samples. As is clear in this figure, the EDMR signals increased under negative $V_{G}$ for all our samples, demonstrating that the defects were distributed in the near-surface $n^{+}$-type region. This is quite reasonable, because ion implantation creates much more damage in the $n^{+}$-type (i.e., high dose) region rather than in the $p$-type region.
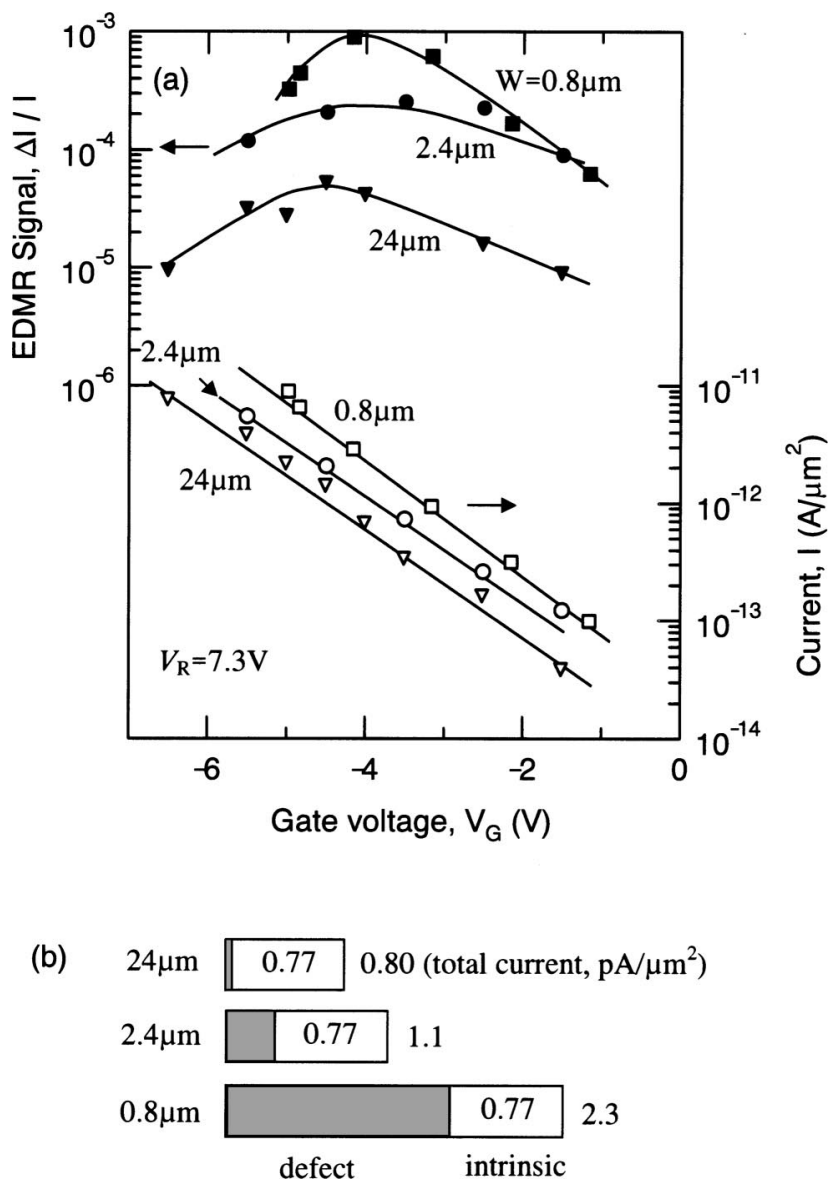

FIG. 4. (a) Gate-bias dependence of the EDMR signal and device current, where the lines are guides to eyes. The signal intensity is calculated from the higher-field-side peak height of signal B. (b) Estimation of the defectinduced current component in a total current at $V_{G}=-4 \mathrm{~V}$.

Together with the EDMR signals, the JLC increased exponentially under negative $V_{G}$. This phenomenon is known as gate-induced drain leakage (GIDL). ${ }^{9}$ It has been concluded that the GIDL originates from band-to-band tunneling and/or trap-assisted tunneling in the gate-induced nearsurface depletion layer. ${ }^{9,10}$ From the results of this study, it is clear that the observed defects are a source of trap-assisted GIDL. Since the GIDL causes non-negligible leakage currents in the channel-OFF (stand-by) state of MOSFETs, it is an important issue for LSI performance. ${ }^{9}$ In particular, the charge-retention time of DRAMs would be considerably reduced by such a GIDL. ${ }^{10}$ Thus, the defects we observed are of special significance in DRAM performance. Similarly to Fig. 3 , a high gate bias caused a decrease in $\Delta I / I$. Since the gate bias increased the electric field in the depletion region (and hence the depletion layer expanded into the $n^{+}$-type region), this behavior can be explained by the occurrence of the direct tunneling.

In Fig. 4(a), it is also notable that $\Delta I / I$ shows a drastic increase as $W$ decreases. In principle, $\Delta I / I$ becomes larger if the defect density of a sample increases. ${ }^{19}$ Therefore, it is reasonable to assume that defect density increases in a narrow-width MOSFET. This is in agreement with the increase in JLC [Fig. 4(a)], which also suggests an increase in the defect density. To clarify this, using the $\Delta I / I$ and $I$ values 


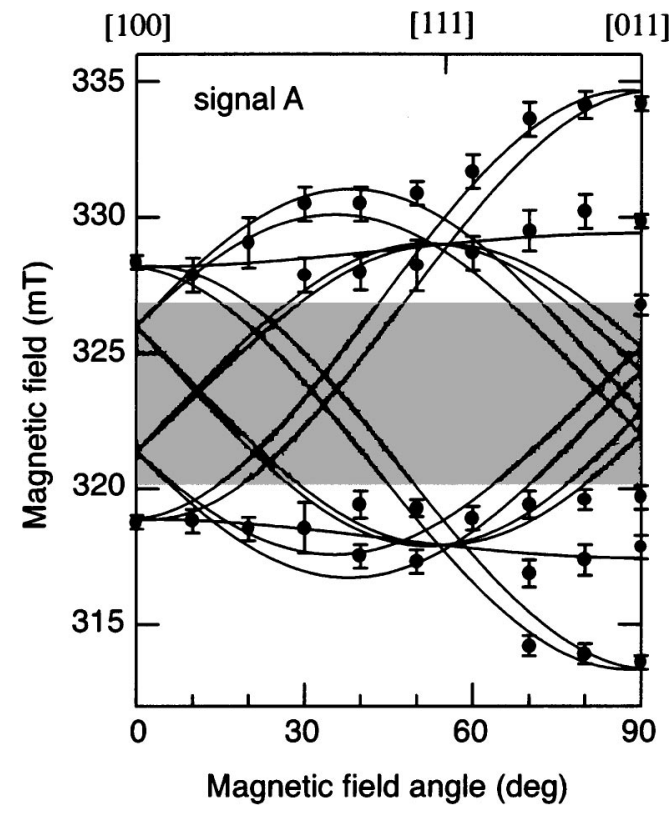

FIG. 5. Angular dependence of signal A. Solid lines represent the best-fit pattern. In the gray area, signal A is not clear due to the overlapping of signal B.

at $V_{G}=-4 \mathrm{~V}$, we estimated the intrinsic and defect-induced components in the total JLC. We assumed that (i) the intrinsic components were constant for the three samples and (ii) $\Delta I / I$ was in proportion to a fraction of the defect-induced components in the total current $I$. The results are shown in Fig. 4(b). This figure indicates that decreasing $W$ causes an increase in the defect-induced component, and therefore an increase in the total JLC. A similar phenomenon was observed in other types of Si LSIs. ${ }^{10,12-14}$ In those experiments, the JLCs increased with the scaling of the device, or with an increase in process-induced stress. Accordingly, this phenomenon has been ascribed to stress effects, such as bandgap narrowing, ${ }^{13}$ formation of dislocations, ${ }^{14}$ or small defects $^{10,12}$ due to stress. Our results suggest that point defects relate to this phenomenon. We speculate that the reason the formation of defects is dependent on a factor of $W$ is connected with the structure of the defects, which is considered subsequently.
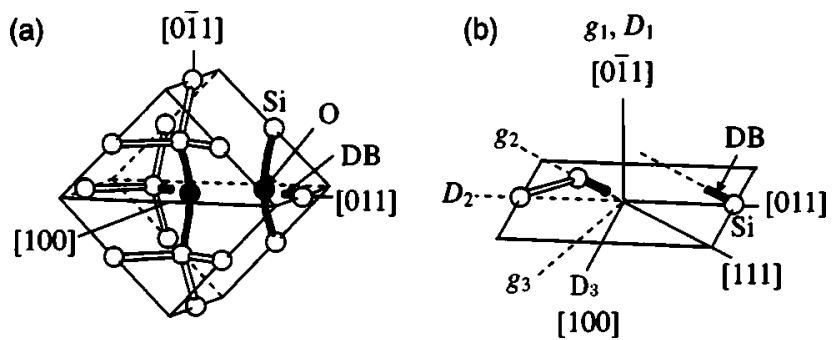

FIG. 6. (a) The $\mathrm{V}_{2}+\mathrm{O}_{2}$ center (the origin of signal A). (b) Defect system common to spin-1 vacancy-oxygen complexes.

\section{B. Origin of signal A (divacancy-oxygen complexes)}

Signal A is a EDMR signal that has not been reported in other EDMR studies. We presume that signal A may have been too weak to be detected in previous studies. This signal splits into multiple lines (Fig. 2) and shows a strong dependence on the magnetic-field (B) orientation. Figure 5 plots the resonant positions of signal $\mathrm{A}$ as a function of the rotation of $\mathbf{B}$ in the $(0 \overline{1} 1)$ plane. One notable characteristic of this pattern is that the ratio between the splitting widths at $\mathbf{B} \|[100]$ and $\mathbf{B} \|[011]$ is exactly 1:2. This strongly suggests that the signal splitting originates from a fine (electron-spinelectron-spin) interaction, which is almost axially symmetric around the [011] direction. Previously, similar fine interactions were reported for a series of spin-1 vacancy-oxygen complexes in radiation-damaged $\mathrm{Si}^{20-23}$ such as $\mathrm{V}_{2}+\mathrm{O}_{2}$ [Fig. 6(a)]. These complexes retain a pair of $\mathrm{Si}$ dangling bonds (DBs) that generate a fine interaction. Thus, we simulated the angular dependence of signal $\mathrm{A}$ by adapting the common defect system to spin-1 complexes [Fig. 6(b)]. The simulation and experimental data appeared to match very well (Fig. 5). Table I summarizes the best-fitting EPR parameters, in addition to the reported values for radiationdamaged $\mathrm{Si}$. The most important parameter is the D (fine interaction) tensor. As can be seen in the table, the principal values of $\mathrm{D}\left(D_{1}\right.$ to $\left.D_{3}\right)$ systematically decrease with the number of $\mathrm{Si}$ vacancies, in accordance with the $R^{-3}$ dependence $(R=$ separation between DBs) of the fine interaction.

TABLE I. EPR parameters for signal A and various spin-1 vacancy-oxygen complexes. The definition of these parameters is shown in Fig. 6(b). The SL1 center corresponds to a photoexcited metastable state (see Ref. 22). The $g_{2}$ axis in this table shows an approximate principal axis of $g_{2}$. The principal D values are expressed in mT. In Fig. 6(b), we simplified the principal axes of $D_{2}$ and $D_{3}$ to be [011] and [100], respectively, however, the reported axes were deviated from these directions.

\begin{tabular}{|c|c|c|c|c|c|c|c|c|c|}
\hline Defect (label) & $R(\mathrm{~nm})$ & $g_{1}$ & $g_{2}$ & $g_{3}$ & $g_{2}$ axis & $D_{1}$ & $D_{2}$ & $D_{3}$ & Ref. \\
\hline Signal A & & 2.008 & 2.002 & 2.010 & {$[\overline{11} \overline{1}]$} & \pm 4.0 & $\mp 7.1$ & \pm 3.1 & Present \\
\hline $\mathrm{V}+\mathrm{O}(\mathrm{SL} 1)$ & 0.38 & 2.0102 & 2.0057 & 2.0075 & {$[0 \overline{1} 1]$} & \pm 21.9 & $\mp 46.8$ & \pm 24.9 & 22 \\
\hline $\mathrm{V}_{2}+\mathrm{O}(\mathrm{A} 14)$ & 0.58 & 2.0090 & 2.0020 & 2.0093 & {$[\overline{1} 1 \overline{1}]$} & \pm 4.2 & $\mp 6.6$ & \pm 2.4 & 20,21 \\
\hline $\mathrm{V}_{2}+\mathrm{O}_{2}(\mathrm{P} 2)$ & 0.58 & 2.0088 & 2.0019 & 2.0099 & {$[\overline{111}]$} & \pm 4.3 & $\mp 7.4$ & \pm 2.9 & 20 \\
\hline $\mathrm{V}_{3}+\mathrm{O}(\mathrm{P} 4)$ & 0.77 & 2.0082 & 2.0036 & 2.0066 & {$[0 \overline{1} 1]$} & \pm 1.4 & $\mp 2.9$ & \pm 1.5 & 20 \\
\hline $\mathrm{V}_{3}+\mathrm{O}_{2}(\mathrm{P} 5)$ & 0.77 & 2.0091 & 2.0050 & 2.0072 & {$[0 \overline{11}]$} & \pm 1.6 & $\mp 3.2$ & \pm 1.6 & 20 \\
\hline $\mathrm{V}_{3}+\mathrm{O}_{3}(\mathrm{~A} 15)$ & 0.77 & 2.0115 & 2.0045 & 2.0085 & {$[0 \overline{1} \overline{1}]$} & \pm 1.9 & $\mp 3.8$ & \pm 1.9 & 20 \\
\hline $\mathrm{V}_{4}(\mathrm{P} 3)$ & 0.96 & 2.0099 & 2.0010 & 2.0102 & {$[\overline{111}]$} & \pm 0.84 & $\mp 1.64$ & \pm 0.80 & 23 \\
\hline
\end{tabular}


Our results are in good agreement with those obtained for the divacancy plus one oxygen atom $\left(\mathrm{V}_{2}+\mathrm{O}\right.$, the $\mathrm{A} 14$ center $)$ or two oxygen atoms $\left(\mathrm{V}_{2}+\mathrm{O}_{2}\right.$, the $\mathrm{P} 2$ center $){ }^{20,21}$ We ruled out the possibility of an intrinsic divacancy $\left(\mathrm{V}_{2}\right)$, because the $\mathrm{V}_{2}$ defect appears as a spin-1/2 center (either G6 or G7 center, if it is positively or negatively charged, respectively). ${ }^{24}$ Therefore, we concluded that signal A originated from a combination of A14 and P2 spectra. The atomic structure of the $\mathrm{V}_{2}$ $+\mathrm{O}$ center is essentially the same as that of $\mathrm{V}_{2}+\mathrm{O}_{2}$ [Fig. 6(a)], except that one $\mathrm{Si}-\mathrm{O}-\mathrm{Si}$ bond is replaced by an elongated $\mathrm{Si}-\mathrm{Si}$ bond. ${ }^{20}$

This atomic structure is also consistent with the $\mathrm{g}$ tensor data. As is seen in Table I, the $\mathrm{g}$ tensor of signal A is nearly axially symmetric around the principal axis of $g_{2}$ (i.e., $g_{1}$ $\approx g_{3}$ ). This indicates that the DB pair is parallel to the $g_{2}$ axis, which is consistent with the model shown in Fig. 6. If the DB pair is not parallel, as is the case for $\mathrm{V}_{1}$ and $\mathrm{V}_{3}$, this axial symmetry is no longer observed. It is also worth noting that the principal $\mathrm{g}$ values of signal $\mathrm{A}$ are quite similar to those of a typical Si DB (the $P_{b}$ and $P_{b 0}$ centers) at the $\mathrm{SiO}_{2} / \mathrm{Si}$ interface. ${ }^{4}$ This also supports our conclusion.

Since a Si DB has an unpaired electron, the generationrecombination process by which a DB captures an electron is spin dependent. This spin-dependent process is only active if the Si DB is located in the depletion layer. Therefore, the $\mathrm{V}_{2}+\mathrm{O}$ and $\mathrm{V}_{2}+\mathrm{O}_{2}$ centers are detectable only when they are located in the depletion layer, which is consistent with our arguments in Sec. A. It should be mentioned that when these Si DBs are located outside the depletion layer, they make no contribution to the JLC as well as to the spindependent process.

\section{Origin of signal B (large Si vacancies)}

Signal B resembles the well-known Si DB signal (g value, $g=2.0055$ ) observed in amorphous $\mathrm{Si}^{25}$ and in $\mathrm{Si}$ crystals damaged by implantation, ${ }^{26}$ irradiation, ${ }^{8}$ or mechanical stress. ${ }^{27}$ The origin of the $g=2.0055$ signal has been generally accepted as isolated Si DBs that are embedded randomly in an amorphous matrix. ${ }^{8,25-27}$ However, this picture does not apply to our $n^{+}-p$ junctions, which were composed entirely of a crystalline Si lattice. Signal B was observed in our local-oxidation-of-Si-isolated $\mathrm{Si} p-n$ junction, which was also composed of crystalline $\mathrm{Si}^{4}{ }^{4}$ Previously, we tentatively assigned the origin of signal $\mathrm{B}$ to an implantation damage center. ${ }^{4}$ However, we were unable to extract spectroscopic information for a weak signal B and were therefore unable to clarify why isotropic Si DBs are embedded in crystalline lattice. In contrast with the previous work, the highintensity spectra in this study [Fig. 7(a)] clearly show that signal B is not isotropic and becomes sharp when $\mathbf{B}$ is aligned in the [100] direction. This anisotropy is clear evidence that the Si DBs were not randomly oriented in our samples. Furthermore, we can exclude the possibility of isolated DBs because their spectra are characterized by split lines, as observed in the $P_{b 0}$ spectrum. ${ }^{4}$ Therefore, we consider that the relevant DBs interact with each other only weakly (i.e., $R$ is large), resulting in the split lines being smeared out by weak fine interactions.

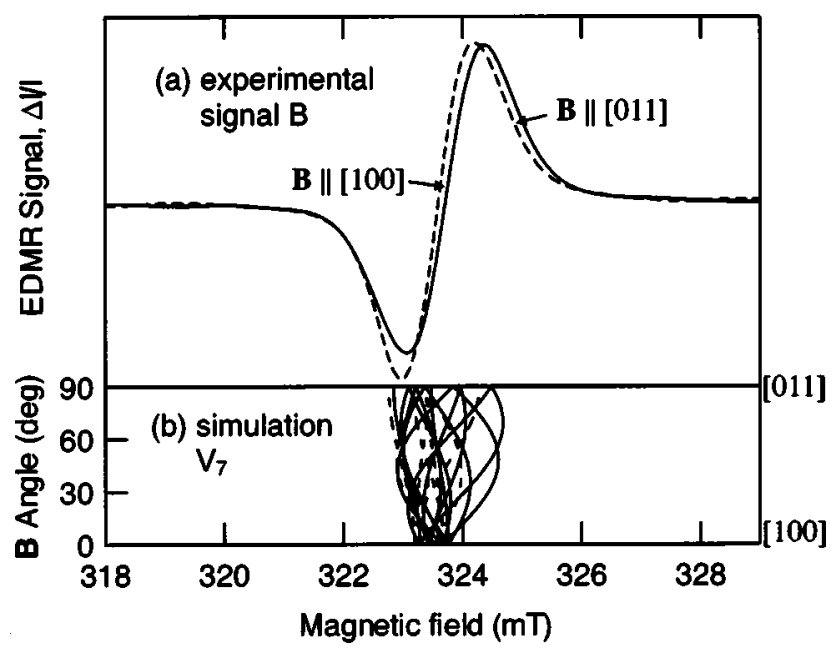

FIG. 7. (a) Angular dependence of signal B. Dashed and solid lines were measured for $\mathbf{B} \|[100]$ and $\mathbf{B} \|[011]$, respectively. (b) Simulated angular dependence of signal $\mathrm{B}$ for the $\mathbf{B}$ rotation in the $(0 \overline{1} 1)$ plane, which was calculated for a model of $\mathrm{V}_{7}+$ a DB pair. Solid and dashed lines correspond to simulations for parallel and nonparallel DB pairs, respectively.

An analogy with signal A indicates that these DBs are probably involved in Si vacancies. In Fig. 8, the maximum fine-splitting widths $\left(D_{\max }\right)$ of the spin-1 $\mathrm{Si}$ vacancies are plotted as a function of $R$. For signal $\mathrm{B}$, the $D_{\max }$ value should be less than the full width at half-maximum of the absorption peak $(=1.5 \mathrm{mT})$. On the basis of the intersection between $1.5 \mathrm{mT}$ and the $R^{-3}$ dependence of $D_{\max }$, we estimated that $R$ is larger than $1.4 \mathrm{~nm}$. This $R$ value approximately corresponds to the length of the $\mathrm{V}_{6}$ to $\mathrm{V}_{7}$ chain. In Fig. 7(b), the angular dependence of signal $B$ was simulated by using a model consisting of $\mathrm{V}_{7}$ plus a DB pair, based on the same defect system used earlier. The $g$ tensor was set to that for either $V_{2}$ and $V_{4}$ (a parallel DB pair) or $V_{1}$ and $V_{3}$ (a nonparallel pair), as shown in Table I. We used D values of $D_{1}= \pm 0.21, D_{2}=\mp 0.37$, and $D_{3}= \pm 0.16 \mathrm{mT}$, which were calculated from their $R^{-3}$ dependence. The two simulation patterns for the different $g$ tensors are superimposed in Fig.

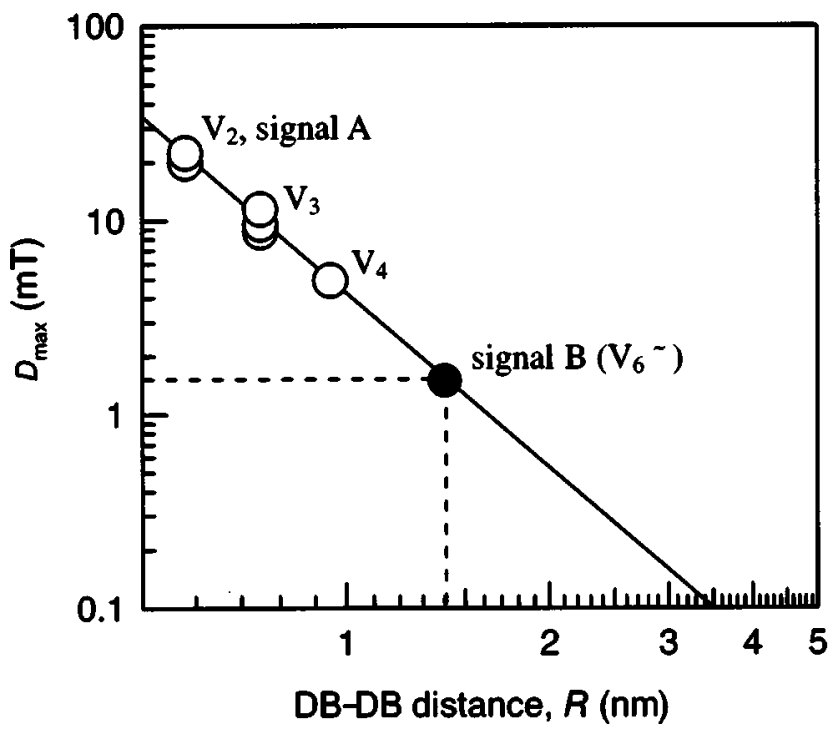

FIG. 8. Maximum fine-splitting width $\left(D_{\max }\right)$ versus DB-DB distance $(R)$. The solid line indicates the $R^{-3}$ dependence. 


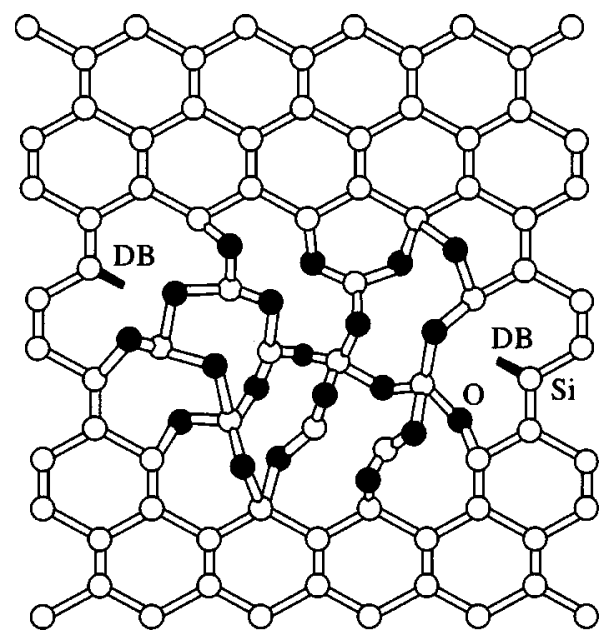

FIG. 9. A defect model for signal B (large Si vacancies including distant $\mathrm{Si}$ DB pairs $(R \geqslant 1.4 \mathrm{~nm})$ and probably oxidized). In this figure, $R$ is $1.6 \mathrm{~nm}$.

7(b). We should mention that this simulation was too simple to account for all actual DB situations. However, it revealed some essential features of signal B. First, due to complicated fine splitting, a complete signal will form a single broad peak for any rotation angle, as observed experimentally. Second, the signal-narrowing effect observed at $\mathbf{B} \|[100]$ has been tentatively reproduced because of the small $D_{3}$ value. Similar results were also obtained when $R$ was increased. Note that since there are a number of possible configurations of DBs and vacancies, the signal broadening proceeds fully, resulting in a complete single line for signal B. Consequently, we propose that signal $\mathrm{B}$ arises from a series of large $\mathrm{Si}$ vacancies $\left(\mathrm{V}_{n}, n \geqslant 6\right)$, which leave weakly interacting $\mathrm{Si}$ DBs $(R$ $\geqslant 1.4 \mathrm{~nm}$ ). These large vacancies are supposedly oxidized during high-temperature processes. Figure 9 illustrates our model for the origin of signal B. We suggest that they are a small type of the oxide precipitates observed by TEM on Czochralski (CZ) Si wafers. ${ }^{28}$ Such oxygen precipitates are formed in the same temperature range used for recovery annealing of ion implantation processes ${ }^{28}$ so similar defects could be formed in Si LSIs.

\section{DISCUSSION: HOW CAN THE VACANCY-TYPE DEFECTS REMAIN IN Si LSIs?}

The microscopic origins of signals A and B have been assigned to small and large Si vacancy-type defects, respectively. Since ion implantation creates a large number of $\mathrm{Si}$ vacancies (and also Si interstitials), this result seems to be reasonable. In our samples, signal B (large vacancies) was always much more dominant than signal $\mathrm{A}\left(\mathrm{V}_{2}+\mathrm{O}\right.$ and $\mathrm{V}_{2}$ $+\mathrm{O}_{2}$ ). This is also reasonable, because larger vacancies show higher thermal stability, as observed in radiationdamaged $\mathrm{Si}^{20,28}$

After implantation, however, our devices were subjected to recovery annealing at $800 \sim 1000^{\circ} \mathrm{C}$, which is much higher than the anneal-out temperature $\left(<400^{\circ} \mathrm{C}\right)$ for $\mathrm{V}_{2}+\mathrm{O}$ and $\mathrm{V}_{2}+\mathrm{O}_{2}$ in bulk Si. ${ }^{20}$ In addition, the $g=2.0055$ signals usually disappear when implantation-damaged $\mathrm{Si}$ wafers are annealed in the appropriate temperature range. ${ }^{26}$ Therefore, $\mathrm{Si}$ vacancy-type defects appear to be much more thermally stable in device structures than in bulk Si crystals.

We propose two physical mechanisms for this phenomenon. One is a mechanical stress due to the structure of the device. As was seen in Fig. 4(a), the STI structure strongly influences the density of residual defects in specific device regions. When MOSFET width $(W)$ is narrowed, compressive stress increases monotonically and becomes several hundred MPa for $W$ in the submicron range. ${ }^{11,12}$ Generally, under such compressive stress, Si vacancies become thermally stable because the tensile stress induced around a $\mathrm{Si}$ vacancy cancels the compressive strain of the Si lattice. ${ }^{29}$ Although this energy gain (=pressure coefficient of the vacancy formation energy, $\Delta E_{f}$ ) has not been determined for vacancy-oxygen complexes, the simplest example of a $\mathrm{Si}$ monovacancy $\left(\mathrm{V}_{1}\right)$ reveals that $\Delta E_{f}=-0.1 \mathrm{eV}$ at a compressive stress of $600 \mathrm{MPa} .{ }^{29}$ Even with this small $\Delta E_{f}, \mathrm{~V}_{1}$ is about three times $\left[\approx \exp \left(\Delta E_{f} / k_{\mathrm{B}} T\right)\right.$, where $k_{\mathrm{B}}$ is the Boltzmann constant] more stable at $T=800^{\circ} \mathrm{C}$ and $600 \mathrm{MPa}$, as compared to a zero-stress situation. It is thus possible that more Si monovacancies remain in a narrower MOSFET, resulting in more $\mathrm{Si}$ vacancy-type defects. The key factor in the effect of stress is how large the $\Delta E_{f}$ value is, which is determined mainly by how large the defect-induced stress is. ${ }^{29}$ If this defect-induced stress is larger for vacancy-oxygen complexes than for monovacancies, the stress effect will become more significant than suggested earlier.

The other important factor is the concentration of oxygen atoms. Studies on oxide precipitates have suggested that once some $\mathrm{Si}$ vacancies are oxidized, they form a thermally stable nucleus for growing large oxide precipitates. ${ }^{28}$ The EPR studies have confirmed more directly that $\mathrm{Si}$ vacancies $\left(\mathrm{V}_{1}\right.$ to $\mathrm{V}_{3}$ ) become increasingly stable when they couple with more oxygen atoms. ${ }^{20}$ Our devices were fabricated in a high-purity epitaxially grown $\mathrm{Si}$ layer, so that the initial oxygen concentration should have been much smaller than that of CZ Si wafers (which involve a supersaturated concentration of oxygen atoms during the crystal growth). In our samples, however, oxygen incorporation could occur in the near-surface regions during oxidation and implantation through scattering oxides. It is thus likely that the oxygen concentration is high only in the near-surface region of the devices and, therefore, that $\mathrm{Si}$ vacancies are thermally stable in such a region. This is consistent with our experimental result that the defects observed were distributed in the nearsurface region (Sec. III A). Recently, a similar phenomenon was found by using positron-annihilation measurements on separation by implanted oxygen (SIMOX) Si wafers. ${ }^{30}$ In this case, vacancy-oxygen complexes were detected in nearsurface regions that contained a two-orders higher concentration of oxygen atoms $\left(10^{20} \mathrm{~cm}^{-3}\right)$ than a typical value for CZ Si wafers. These complexes were stable even in processes at $1300^{\circ} \mathrm{C}$. Interestingly, their sizes were tentatively estimated to be $\mathrm{V}_{1} \sim \mathrm{V}_{2}\left(=\mathrm{V}_{2}+\right.$ oxygen atoms) or larger than $\mathrm{V}_{6}$, which is quite consistent with our microscopic data. 
In our samples, only $\mathrm{Si}$ vacancies were observed and no $\mathrm{Si}$ interstitials (self-interstitials) were found. Basically, ion implantation creates Frenkel pairs of vacancies and interstitials. The question then arises: Why were the self-interstitials missing? We speculate as follows: (i) self-interstitials are much more mobile than vacancies, ${ }^{31}$ which means they may be captured by gettering centers outside the device region; (ii) self-interstitials are not stabilized by coupling with oxygen atoms, but are strongly stabilized by boron atoms in the $p$-type $\mathrm{Si}^{31}$ which means they may not be thermally stable in our $n$-channel MOSFETs. For these reasons, Si vacancies may be the dominant defects in our devices.

\section{SUMMARY}

We carried out EDMR studies on the microscopic structure of the ion-implantation damage centers that remain in as-fabricated Si LSIs. Using special EDMR techniques that were extremely sensitive to defects, we detected two types of implantation damage defects in the source/drain $\left(n^{+}\right.$-type) region of 0.25 - $\mu \mathrm{m}$-gate-length $n$-channel MOSFETs on LSIs. One defect was identified as a spin-1 $\mathrm{Si}$ DB pair $(R$ $\approx 0.6 \mathrm{~nm})$ in a divacancy-oxygen complex $\left(\mathrm{V}_{2}+\mathrm{O}\right.$ and $\mathrm{V}_{2}$ $+\mathrm{O}_{2}$ ). The other was attributed to a series of larger $\mathrm{Si}$ vacancies including distant Si DBs $(R \geqslant 1.4 \mathrm{~nm})$. These large vacancies may be oxidized to form small types of oxide precipitates. Our experiments suggested that these vacancy-type defects are thermally stable in scaled-down devices, as compared to those in bulk Si crystals. The physical origins of this enhanced stability were attributed to the following causes: (i) internal lattice stress due to the structure of devices (in particular, STI structures) raises the stability of defects by canceling the lattice stress caused by defect-induced stress, and that (ii) a high concentration of oxygen atoms in the nearsurface regions of devices strongly stabilizes the vacancytype defects. It is also notable that these defects relate directly to the GIDL current of MOSFETs, which is an important issue for the performance of Si LSIs.
${ }^{1}$ H. Cerva, M. Engelhardt, M. Hierlemann, M. Pölzl, and T. Thenikl, Physica B 308-310, 13 (2001).

${ }^{2}$ D. J. Lepine, Phys. Rev. B 6, 436 (1972).

${ }^{3}$ S. Greulich-Weber, Mater. Sci. Forum 143-147, 1337 (1994).

${ }^{4}$ T. Wimbauer, K. Ito, Y. Mochizuki, Miyazaki, Kitano, M. S. Brandt, and M. Stutzmann, Appl. Phys. Lett. 76, 2280 (2000).

${ }^{5}$ T. Umeda, Y. Mochizuki, K. Okonogi, and K. Hamada, Physica B 308310, 1169 (2001).

${ }^{6}$ G. D. Watkins, Radiation Damage and Defects in Semiconductors, edited by J. E. Whitehouse (Institute of Physics Bristol, 1973), pp. 228-237.

${ }^{7}$ F. Rong, E. H. Poindexter, M. Harmatz, W. R. Buchwald, and G. J. Gerardi, Solid State Commun. 76, 1083 (1990).

${ }^{8}$ M. A. Jupina and P. M. Lenahan, IEEE Trans. Nucl. Sci. 36, 1800 (1989).

${ }^{9}$ T. Y. Chan, J. Chen, P. K. Ko, and C. Hu, Tech. Dig. - Int. Electron Devices Meet. 1987, 718.

${ }^{10}$ J. A. Mandelman, R. H. Dennard, G. B. Bronner, J. K. DeBrosse, R. Divakaruni, Y. Li, and C. J. Radens, IBM J. Res. Dev. 46, 187 (2002).

${ }^{11}$ K. F. Dombrowski et al., Tech. Dig. - Int. Electron Devices Meet. 1999, 357.

${ }^{12}$ A. Steegen, A. Lauwers, M. de Potter, G. Badenes, R. Rooyackers, and M. Maex, VLSI Tech. Dig. of Technical Papers, 2000, 180.

${ }^{13}$ P. Smeys, P. B. Griffin, Z. U. Rek, I. D. Wolf, and K. C. Saraswat, IEEE Trans. Electron Devices 46, 1245 (1999).

${ }^{14}$ D. Ha, C. Cho, D. Shin, G.-H. Koh, T.-Y. Chung, and K. Kim, IEEE Trans. Electron Devices 46, 940 (1999).

${ }^{15}$ A. Toda, N. Ikarashi, H. Ono, S. Ito, T. Toda, and K. Imai, Appl. Phys. Lett. 80, 2278 (2002).

${ }^{16}$ S. M. Sze, Physics of Semiconductor Devices, 2nd ed. (Wiley, New York, 1981), Chap. 2.

${ }^{17}$ F. C. Rong, W. R. Buchwald, E. H. Poindexter, W. L. Warren, and D. J. Keeble, Solid-State Electron. 34, 835 (1991).

${ }^{18}$ G. Vincent, A. Chantre, and D. Bois, J. Appl. Phys. 50, 5484 (1979).

${ }^{19}$ K. Lips, C. Lerner, and W. Fuhs, J. Non-Cryst. Solids 198-200, 267 (1996).

${ }^{20}$ Y.-H. Lee and J. W. Corbett, Phys. Rev. B 13, 2653 (1976).

${ }^{21}$ E. G. Sieverts and J. W. Corbett, Solid State Commun. 43, 41 (1982).

${ }^{22}$ K. L. Brower, Phys. Rev. B 4, 1968 (1971).

${ }^{23}$ W. Jung and G. S. Newell, Phys. Rev. 132, 648 (1963).

${ }^{24}$ G. D. Watkins and J. W. Corbett, Phys. Rev. A 138, 543 (1965).

${ }^{25}$ T. Umeda, S. Yamasaki, J. Isoya, and K. Tanaka, Phys. Rev. B 59, 4849 (1999).

${ }^{26}$ K. L. Brower and W. Beezhold, J. Appl. Phys. 43, 3499 (1972).

${ }^{27}$ B. P. Lemke and D. Haneman, Phys. Rev. B 17, 1893 (1978).

${ }^{28}$ W. A. Tiller, S. Hahn, and F. A. Ponce, J. Appl. Phys. 59, 3255 (1986).

${ }^{29}$ O. Sugino and A. Oshiyama, Phys. Rev. B 46, 12335 (1992).

${ }^{30}$ A. Uedono, Z. Q. Chen, A. Ogura, H. Ono, R. Suzuki, T. Ohdaira, and T. Mikado, J. Appl. Phys. 90, 6026 (2001).

${ }^{31}$ S. C. Jain, W. Schoenmaker, R. Lindsay, P. A. Stolk, S. Decoutere, M. Willander, and H. E. Maes, J. Appl. Phys. 91, 8919 (2002). 